\title{
A comparison of delayed versus immediate reconstruction following lower-extremity sarcoma resection
}

\author{
Sarah Zhou ${ }^{1}$, Alain J Azzi ${ }^{2}$, Tyler Safran ${ }^{2}$, Teanoosh Zadeh ${ }^{2}$ \\ ${ }^{1}$ McGill University Faculty of Medicine, Montreal, QC; ${ }^{2}$ Division of Plastic and Reconstructive Surgery, McGill University Health Center, \\ Montreal, QC, Canada
}

Background Identifying patients who may be at high risk for wound complications postsarcoma resection and reconstruction is essential for improving functional outcomes and quality of life. Currently, the effect of timing on sarcoma reconstruction has been poorly investigated. The purpose of this study was to compare outcomes of delayed and immediate reconstruction in the setting of sarcoma resection requiring flap reconstruction in the lower extremity.

Methods A retrospective review of the senior author's sarcoma reconstruction patients from January 2005 to July 2017 was completed. All patients undergoing flap reconstruction of the lower extremity were included. Complications in the early postoperative period were compared between delayed and immediate reconstructive procedures.

Results A total of 32 patients (7 delayed, 25 immediate) were included in this study. There was a significantly increased rate of overall complications ( $100 \%$ vs. $28.0 \%, P=0.001)$ and rate of hematomas $(28.6 \%$ vs. $0.0 \%, P=0.042)$ in the delayed reconstruction group. Other complications including dehiscence, seroma, infection, venous thrombosis, and total/partial flap loss were also increased in the delayed reconstruction group, but this was not considered to be significant.

Conclusions This study suggests that delayed reconstruction following sarcoma resection of the lower extremity had a higher incidence of overall complications and hematoma formation. We emphasize the importance of early plastic and reconstructive surgeon referral and the necessity to closely monitor delayed reconstruction patients for complications.

Keywords Sarcoma / Lower extremity / Reconstructive surgery / Surgical flaps / Time factors
Correspondence: Alain J Azzi Division of Plastic and Reconstructive Surgery, McGill University Health Center, 1650 Cedar Ave., Montreal, QC H3G 1A4, Canada

Tel: +1-514-934-1934 E-mail: alain.azzi@mail.mcgill.ca
This article was presented as a free paper at the 36th International Meeting of the Korean Society for Aesthetic Plastic Surgery on April 8, 2018, in Seoul, Korea.

Received: May 28, 2019 • Revised: November 20, 2019• Accepted: November 28, 2019

pISSN: 2234-6163・ elSSN: 2234-6171 • https://doi.org/10.5999/aps.2019.00780 • Arch Plast Surg 2020;47:49-53

\section{INTRODUCTION}

Sarcomas are a rare form of cancer that account for less than $1 \%$ of new adult malignancies [1]. Historically, the treatment of extremity sarcomas was amputation of the affected limb [2].
However, in order to improve quality of life and functional outcomes, more recent management emphasizes that adequate control of the disease can be achieved through limb-sparing surgery [3]. For appropriately selected patients, chemotherapy and radiation have also demonstrated an overall survival advantage 
[4].

The NCCN (National Comprehensive Cancer Network) guidelines state that definitive surgical resection with negative margins is the mainstay for curative treatment [5]. In cases of extensive resection, primary closure is seldom possible, and may require locoregional or free flap reconstruction [6]. With limbsparing surgical excision, the benefit of increased functionality comes at a cost of increased rates of wound complications [3]. In order to limit the frequency of postoperative wound complications, it is imperative to identify high-risk patients who may require more aggressive monitoring and wound care.

Previously, a retrospective review examining the predictive factors of wound complications post-sarcoma reconstruction determined that the most predictive factor of complications was whether the procedure was delayed or immediate [2]. Although the effect of timing of sarcoma reconstruction has been poorly investigated, several other retrospective studies correspondingly found higher rates of major wound complications when reconstruction was not done promptly after oncologic surgery $[2,3$, $6,7]$. The purpose of this study was to compare outcomes of delayed and immediate reconstruction in the setting of sarcoma resection requiring flap reconstruction in the lower extremity.

\section{METHODS}

After approval from the Institutional Research Ethics Board (approval number: 2017-3146), a retrospective review of all patients treated by the senior author for sarcoma reconstruction from January 2005 to July 2017 was performed. Two independent reviewers collected data from a combination of electronic and paper medical records. All patients who underwent vascularized tissue reconstruction using free flaps or pedicled flaps post-sarcoma resection were included. Patients who were lost to follow-up ( $<3$ months) or transferred to another institution were excluded from the study.

All lower extremity sarcomas requiring flap reconstructions were included, irrespective of tumor histopathology. Sarcoma resection was performed by the institution's orthopedic surgery team. Patients were classified as delayed or immediate depending on whether the reconstructive surgery occurred in the same procedure as the tumor resection. Demographic information, patient comorbidities and tumor characteristics were collected. Information on neoadjuvant and adjuvant chemotherapy and radiotherapy treatments were also recorded. The primary outcome of interest was early postoperative wound complications including: infection, dehiscence, hematoma, seroma, arterial thrombosis, venous thrombosis, and partial/total flap loss.

The outcomes between delayed and immediate reconstruc- tion groups were compared using Fisher's exact test (categorical variables). For continuous variables (age, tumor size, and tumor depth), delayed and immediate reconstruction groups were compared using an unpaired t-test. Statistical significance was set at a P-value of 0.05 . Means and frequencies were calculated for continuous variables and categorical data, respectively. All statistical analyses were carried out using GraphPad Prism 6 software (La Jolla, CA, USA).

\section{RESULTS}

From January 2005 to July 2017, a total of 32 patients underwent flap (free or pedicled) reconstruction of the lower extremity at our sarcoma center. Seven patients were included in the delayed reconstruction group and 25 patients were included in the immediate reconstruction group. For patients who had a delay in reconstruction, the average delay between resection and reconstruction was 3.2 weeks, with a standard deviation of 1.6 weeks. Patients had a minimum follow-up time of 3 months.

\section{Patient and tumor demographics}

Patient demographics, pathological diagnosis, tumor size, and neo-/adjuvant therapy are described in Table 1 . There was an increased incidence of hypertension in the delayed reconstruction group $(57.1 \%$ vs. $16.0 \%, \mathrm{P}=0.047)$. In the immediate reconstruction group, there was an increased incidence of neoadjuvant radiotherapy ( $64.0 \%$ vs. $14.3 \%, \mathrm{P}=0.033)$. There were no other significant differences between the two groups. A breakdown of the type of flap reconstructions is provided in Table 2 .

\section{Complications}

In order to assess the outcomes of reconstructive surgery, the wound and microvascular complications between delayed and immediate procedures are compared in Table 3. Overall, all patients $(7 / 7,100 \%)$ who underwent delayed reconstruction procedures had at least one complication. The most common postoperative complication was infection $(5 / 7,71.4 \%)$. Other wound complications included hematoma $(2 / 7,28.6 \%)$, dehiscence $(1 / 7,14.3 \%)$, and seroma $(1 / 7,14.3 \%)$. Microvascular complications in the delayed reconstruction group included partial flap loss $(2 / 7,28.6 \%)$, total flap loss $(1 / 7,14.3 \%)$ and venous thrombosis $(1 / 7,14.3 \%)$.

In the immediate reconstruction group, seven patients had at least one complication $(7 / 32,28.0 \%)$. the most common complication was also infection $(7 / 32,28.0 \%)$. Other wound complications in the immediate reconstruction group included seroma $(2 / 32,8.0 \%)$ and dehiscence $(1 / 32,4.0 \%)$. Microvascular 
Table 1. Patient and tumor demographics

\begin{tabular}{|c|c|c|c|c|}
\hline Variable & $\begin{array}{l}\text { Delayed } \\
(n=7)\end{array}$ & $\begin{array}{c}\text { Immediate } \\
(n=25)\end{array}$ & $\begin{array}{l}\text { Overall } \\
(n=32)\end{array}$ & P-value ${ }^{a)}$ \\
\hline \multicolumn{5}{|l|}{ Patient demographics } \\
\hline Age (yr) & $64.0 \pm 19.5$ & $47.5 \pm 19.9$ & $51.1 \pm 20.7$ & 0.061 \\
\hline Female sex & $4(57.1)$ & $8(40.0)$ & $14(43.8)$ & 0.670 \\
\hline Diabetes & $0(0.0)$ & $3(12.0)$ & $3(9.4)$ & $\geq 0.999$ \\
\hline Hypertension & & & & $0.047^{e)}$ \\
\hline Dyslipidemia & $3(4$ & & & 0.157 \\
\hline Smokin & $2(2$ & 4 & 6 & 590 \\
\hline Peripheral & & & & \\
\hline \multicolumn{5}{|l|}{ Pathological diagnosis } \\
\hline Soft tissue sarcoma & $3(42.9)$ & 19( & 22( & 0.166 \\
\hline Bony & & & & \\
\hline \multicolumn{5}{|l|}{ Tumor characteristics } \\
\hline Size $(\mathrm{cm})^{b)}$ & $15.7=$ & c) & $10.9 \pm 10.1^{c)}$ & 0.059 \\
\hline Dept & $8.1 \pm 3.7^{\mathrm{d})}$ & $5.5 \pm 4.5^{d)}$ & $6.0 \pm 4.4^{d)}$ & 0.248 \\
\hline Bone resection & $4(57.1)$ & $5(20.0)$ & $9(28.1)$ & 0.076 \\
\hline \multicolumn{5}{|l|}{ Neo/adjuvant therapy } \\
\hline Neoadjuvant chemotherapy & $2(28$ & 4( & $6(1$ & 0.590 \\
\hline Adjuvant chemotherapy & $4(5)$ & $5(2$ & $9(2$ & 0.076 \\
\hline Neoadjuvant radiotherapy & $1(14.3)$ & $16(64.0)$ & $17(5$ & $0.033^{\mathrm{e})}$ \\
\hline Adjuvant radiotherapy & $3(42.9)$ & $9(36.0)$ & $12(37.5)$ & $\geq 0.999$ \\
\hline \multicolumn{5}{|c|}{ 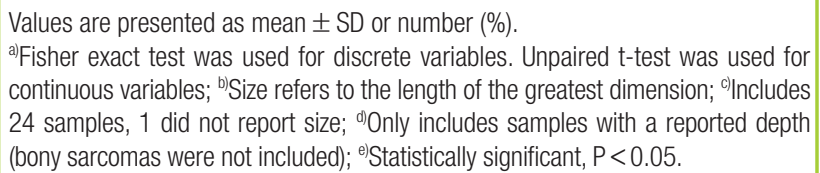 } \\
\hline
\end{tabular}

\section{Table 2. Flap selection}

\begin{tabular}{|lcc|}
\hline Flap & $\begin{array}{c}\text { Delayed } \\
(\mathbf{n}=\mathbf{7})\end{array}$ & $\begin{array}{c}\text { Immediate } \\
(\mathbf{n}=\mathbf{2 5})\end{array}$ \\
\hline Free flaps & 0 & 1 \\
ALT & 0 & 1 \\
Latissimus dorsi & 0 & 6 \\
FRFF & 1 & 0 \\
VRAM & & \\
Pedicled flaps (myocutaneous) & 1 & 9 \\
Gastrocnemius & 1 & 0 \\
Rectus femoris & 2 & 2 \\
Rectus abdominis & 1 & 0 \\
Vastus lateralis & 1 & 1 \\
Gracilis/Adductor longus & & \\
Pedicled flaps (axial fasciocutaneous) & 0 & 2 \\
Reverse superficial sural artery & 0 & 1 \\
Internal pudendal artery & 0 & 2 \\
Other & & \\
\hline ALT, anterolateral thigh flap; FRFF, free radial forearm flap; VRAM, vertical rectus \\
abdominis flap.
\end{tabular}

complications included total flap loss $(1 / 32,4.0 \%)$ and partial flap loss $(1 / 32,4.0 \%)$.

Overall, a significant increase in overall complication rate ( $100 \%$ vs. $28.0 \%, \mathrm{P}=0.001)$ and hematoma $(28.6 \%$ vs. $0.0 \%$, $\mathrm{P}=0.042)$ was seen in the delayed reconstruction group.

\section{Table 3. Complications}

\begin{tabular}{|c|c|c|c|}
\hline Complication & $\begin{array}{c}\text { Delayed } \\
(n=7)\end{array}$ & $\begin{array}{l}\text { Immediate } \\
(\mathrm{n}=25)\end{array}$ & P-value \\
\hline \multicolumn{4}{|l|}{ Wound complications } \\
\hline Dehiscence & $1(14.3)$ & $1(4.0)$ & 0.395 \\
\hline Hematoma & $2(28.6)$ & 0 & $0.042^{b}$ \\
\hline Seroma & $1(14.3)$ & $2(8.0)$ & 0.536 \\
\hline Infection & $5(71.4)$ & $7(28.0)$ & 0.074 \\
\hline \multicolumn{4}{|c|}{ Microvascular complications } \\
\hline Venous thrombosis & $1(14.3)$ & 0 & 0.219 \\
\hline Arterial thrombosis & 0 & 0 & $\geq 0.999$ \\
\hline Total flap lossª) & $1(14.3)$ & $1(4.0)$ & 0.395 \\
\hline Partial flap loss ${ }^{a)}$ & $2(28.6)$ & $1(4.0)$ & 0.113 \\
\hline Overall complications & $7(100)$ & $7(28.0)$ & $0.001^{b}$ \\
\hline
\end{tabular}

\section{DISCUSSION}

In order to improve functional outcomes, limb-salvage surgery has become the standard treatment for sarcomas of the extremity. The rate of complications in sarcoma procedures is between $30 \%$ and $40 \%$, depending on supplemental neoadjuvant or adjuvant therapy $[2,8,9]$. In particular, lower-extremity reconstruction has a tendency to have increased rates of complication and flap failure due to the scarcity of local soft tissue and blood supply [10-12]. Thus, identifying the procedural factors that contribute to high complication rates is important to help direct treatment guidelines. Our study aimed to investigate the effect of the timing of flap reconstruction, specifically in sarcomas of the lower extremity.

This study highlights that delayed flap reconstruction of the lower extremity is associated with an increased rate of wound complications compared to immediate reconstruction (100\% vs. $28.0 \%, \mathrm{P}=0.001)$. While our study focuses specifically on the lower extremity, the results remain consistent with the available literature, which comprised of small retrospective cohort studies $[2,3,6,7]$. Sanniec et al. [7] found that delaying reconstruction after sarcoma resection led to an increased number of infections requiring antibiotics ( $47.1 \%$ vs. $9.4 \%, \mathrm{P}=0.0016)$. The authors postulate that immediate reconstruction of sarcoma defects have superior healing outcomes due to the introduction of healthy tissue to increase circulation and bacterial clearance [7]. When delayed sarcoma reconstruction is necessary, they suggest that prophylactic antibiotics are to be considered as standard treatment. While we also report an increased rate of infection with delayed reconstruction ( $71.4 \%$ vs. $28.0 \%)$, the difference was not found to be significant $(\mathrm{P}=0.074)$.

In the current study, delayed reconstruction was associated with a significantly increased incidence of hematoma ( $28.6 \%$ vs. 
$0 \%, \mathrm{P}=0.0423)$. Published studies have not previously observed this outcome. We hypothesize that delaying reconstruction may expose tissues and vessels that, even with careful dressing changes and wound care, may leave vessels susceptible to damage and/or desiccation. These open wounds might have also been infected, thus exposing the tissue to inflammation and altered hemostasis. Moreover, additional debridement due to the delayed nature of the reconstruction might have also increased the risk of hematoma in these cases. Another contributing factor may be the significantly increased rate of hypertension present in the delayed reconstruction group, as there is evidence of correlation between perioperative blood pressure and hematoma development [13]. It is possible that this may have contributed to the observed increased rates of hematoma.

When comparing concurrent treatment, patients in the immediate reconstruction group had a significantly increased number of patients who underwent neoadjuvant radiotherapy. Although useful to diminish tumor sizes, it has been well-established that the cytotoxic effects of radiotherapy create an unfavorable environment for reconstructive surgery and wound healing [14]. This is the second time that our group has not observed an increase in postoperative complications following neoadjuvant radiation therapy [15].

Our sarcoma center incorporates treatment involving a multidisciplinary team of pathologists, radiologists, oncologists and surgeons. Outcomes of local control and survival are shown to be improved when treated at specialized sarcoma centers, which is defined as a hospital with a multidisciplinary team for managing sarcomas [16]. Marre et al. [3] found that late reconstructive management results in higher complication rates and can lead to subsequent worsening of functional outcomes and quality of life. They recommend that reconstructive surgeons are to be involved with the interdisciplinary team and decision-making process from day one of sarcoma management [3].

The current study suggests a similar conclusion. In the delayed reconstruction group, plastic and reconstructive surgeons were only consulted following sarcoma resection. Patients are all seen by a surgical oncologist. If a large defect is anticipated, the patient is seen by a plastic and reconstructive surgeon pre-operatively for planning. However, if primary closure is anticipated, the patient is not seen by a reconstructive surgeon. All the patients in the delayed reconstruction group either had a larger than expected resection or a failed attempt at primary closure (dehiscence). Dehiscence was caused by infection (3 patients), seroma ( 1 patient), hematoma ( 1 patient) and unknown causes (2 patients). In contrast, plastic and reconstructive surgeons had already been involved in the patient care and management in the immediate reconstruction group. Early preoperative involve- ment of the plastic and reconstructive surgeon could potentially prevent delayed reconstruction by providing the most appropriate alternative to primary closure in these lower extremity resections that are at high risk of complications. Although primary closure is possible in certain cases, the reconstructive surgeon can provide a robust and reliable reconstructive option (e.g., vascularized tissue) that is more resistant to the inevitable complications in lower extremity sarcoma resections. Communication between the reconstructive surgeon and the remainder of the team is of utmost importance for the patient. Proper planning and clear communication can favor immediate reconstruction, obviating the potential risks with delayed procedures and the risks associated with general anesthesia.

The current study is not without limitations. As a single-center retrospective study with a relatively small number of patients, further studies are needed for proof of generalizability and causality. Moreover, delayed reconstructions may have been unavoidable due to longer and/or more complicated resections, predisposing these patients to higher complication rates. The patient population in the delayed reconstruction group may have also been at greater risk of complications after reconstruction, irrespective of the timing of the operation (plastic surgery was only consulted after a failed attempt at primary closure attempt). Given the retrospective nature of this study, we acknowledge that we were not able to collect an even sample size and that inevitably, selection bias may have occurred. Moreover, investigating the correlation between complication rate and days of delay would have been of interest, however, it was not possible with our data given that $100 \%$ of the patients in the delayed group had complications.

Future prospective and randomized controlled trials will hopefully evaluate the impact of delaying reconstruction, while controlling for the above confounding factors. While our results concur with previously published studies, it would be imperative to study the results of more centers with a prospective design and larger sample size.

In conclusion, this study suggests that delayed reconstruction following sarcoma resection of the lower extremity had a higher incidence of overall complications and hematoma formation. We emphasize the importance of early plastic and reconstructive surgeon referral and the necessity to closely monitor delayed reconstruction patients for complications.

\section{NOTES}

\section{Conflict of interest}

No potential conflict of interest relevant to this article was reported. 


\section{Ethical approval}

The study was approved by the Institutional Review Board of McGill University Health Center (IRB No. 2017-3146) and performed in accordance with the principles of the Declaration of Helsinki.

\section{Author contribution}

Conceptualization: Azzi AJ, Zadeh T. Data curation: Zhou S, Safran T. Methodology: Azzi AJ, Zadeh T. Project administration: all authors. Visualization: all authors. Writing original draft: Zhou S, Azzi AJ. Writing- review \& editing: all authors. Approval of final manuscript: all authors.

\section{ORCID}

Sarah Zhou https://orcid.org/0000-0002-9351-8487

Alain J Azzi https://orcid.org/0000-0003-3250-5305

Tyler Safran https://orcid.org/0000-0002-0795-3109

\section{REFERENCES}

1. Clark MA, Fisher C, Judson I, et al. Soft-tissue sarcomas in adults. N Engl J Med 2005;353:701-11.

2. Sanniec KJ, Swanson S, Casey WJ 3rd, et al. Predictive factors of wound complications after sarcoma resection requiring plastic surgeon involvement. Ann Plast Surg 2013;71: 283-5.

3. Marre D, Buendia J, Hontanilla B. Complications following reconstruction of soft-tissue sarcoma: importance of early participation of the plastic surgeon. Ann Plast Surg 2012;69: 73-8.

4. Spierer MM, Alektiar KM, Zelefsky MJ, et al. Tolerance of tissue transfers to adjuvant radiation therapy in primary soft tissue sarcoma of the extremity. Int J Radiat Oncol Biol Phys 2003;56:1112-6.

5. von Mehren M, Randall RL, Benjamin RS, et al. Soft tissue sarcoma, version 2.2016, NCCN clinical practice guidelines in oncology. J Natl Compr Canc Netw 2016;14:758-86.

6. Reece GP, Schusterman MA, Pollock RE, et al. Immediate versus delayed free-tissue transfer salvage of the lower extremity in soft tissue sarcoma patients. Ann Surg Oncol 1994;1:11-7.

7. Sanniec KJ, Velazco CS, Bryant LA, et al. Immediate versus delayed sarcoma reconstruction: impact on outcomes. Sarcoma 2016;2016:7972318.

8. LeBrun DG, Guttmann DM, Shabason JE, et al. Predictors of wound complications following radiation and surgical resection of soft tissue sarcomas. Sarcoma 2017;2017:5465130.

9. Moore J, Isler M, Barry J, et al. Major wound complication risk factors following soft tissue sarcoma resection. Eur J Surg Oncol 2014;40:1671-6.

10. Slump J, Hofer SOP, Ferguson PC, et al. Flap choice does not affect complication rates or functional outcomes following extremity soft tissue sarcoma reconstruction. J Plast Reconstr Aesthet Surg 2018;71:989-96.

11. Culliford AT 4th, Spector J, Blank A, et al. The fate of lower extremities with failed free flaps: a single institution's experience over 25 years. Ann Plast Surg 2007;59:18-21.

12. Kang MJ, Chung $\mathrm{CH}$, Chang YJ, et al. Reconstruction of the lower extremity using free flaps. Arch Plast Surg 2013;40: 575-83.

13. Farkas JP, Kenkel JM, Hatef DA, et al. The effect of blood pressure on hematoma formation with perioperative Lovenox in excisional body contouring surgery. Aesthet Surg J 2007;27:589-93.

14. Haubner F, Ohmann E, Pohl F, et al. Wound healing after radiation therapy: review of the literature. Radiat Oncol 2012; $7: 162$.

15. Azzi AJ, Zhou S, Safran T, et al. Vascularized tissue reconstruction in previously irradiated sarcoma defects. Ann Plast Surg 2019;82:89-92.

16. Bhangu AA, Beard JA, Grimer RJ. Should soft tissue sarcomas be treated at a specialist centre? Sarcoma 2004;8:1-6. 